ARTICLE

\title{
Ultrafast spin exchange-coupling torque via photo-excited charge-transfer processes
}

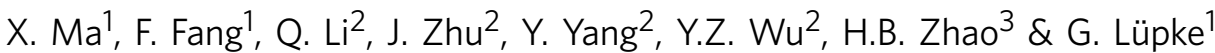

Optical control of spin is of central importance in the research of ultrafast spintronic devices utilizing spin dynamics at short time scales. Recently developed optical approaches such as ultrafast demagnetization, spin-transfer and spin-orbit torques open new pathways to manipulate spin through its interaction with photon, orbit, charge or phonon. However, these processes are limited by either the long thermal recovery time or the low-temperature requirement. Here we experimentally demonstrate ultrafast coherent spin precession via optical charge-transfer processes in the exchange-coupled $\mathrm{Fe} / \mathrm{CoO}$ system at room temperature. The efficiency of spin precession excitation is significantly higher and the recovery time of the exchange-coupling torque is much shorter than for the demagnetization procedure, which is desirable for fast switching. The exchange coupling is a key issue in spin valves and tunnelling junctions, and hence our findings will help promote the development of exchange-coupled device concepts for ultrafast coherent spin manipulation.

\footnotetext{
${ }^{1}$ Department of Applied Science, College of William and Mary, 251 Jamestown Road, Williamsburg, Virginia 23187, USA. ${ }^{2}$ State Key Laboratory of Surface Physics, Department of Physics, and Collaborative Innovation Center of Advanced Microstructures, Fudan University, Shanghai 200433, China. ${ }^{3}$ Key Laboratory of Micro and Nano Photonic Structures (Ministry of Education), Department of Optical Science and Engineering, Fudan University, Shanghai, 200433, China. Correspondence and requests for materials should be addressed to H.B.Z. (email: hbzhao@fudan.edu.cn) or to G.L. (email: gxluep@wm.edu).
} 
C ontrol of coherent spin precession in ferromagnets is currently a popular topic due to its importance in magnetic recording and spintronic devices ${ }^{1-6}$. The search for non-thermal excitation mechanisms motivates extensive research to overcome the disadvantages of thermal ones. The main idea is to utilize the interaction between magnetization and photo-excited carriers that are selectively optical pumped, where the recombination time of photocarriers is much shorter than the heat diffusion process. A promising approach is through ferromagnetic-antiferromagnetic (FM-AFM) exchange coupling, as small modulation of the exchange-coupling strength might lead to notable changes in magnetic properties ${ }^{7,8}$. Recent studies demonstrated that short laser pulses can introduce non-thermal spin reorientation and dynamics in AFM materials much faster than in FM materials ${ }^{9,10}$. But the question is still open whether it is possible to drive FM magnetization at the speed of AFM materials through FM-AFM exchange across heterostructure interface.

In this article, optical excitation of spin precession is investigated in $\mathrm{Fe} / \mathrm{CoO}$ exchange-coupled heterostructure with time-resolved magneto-optic Kerr effect (TRMOKE). Photoexcited charge-transfer processes in AFM CoO layer create a strong transient exchange-coupling torque $\tau_{\mathrm{ex}}(t)$ on FM Fe layer through FM-AFM exchange coupling. The efficiency of spin precession excitation is significantly higher and the recovery is notably faster than the demagnetization procedure. The precession amplitude peaks around room temperature and with external magnetic field competitive to the magnetic anisotropy field, indicating that this efficient excitation mechanism originates from the modulation of the uniaxial magnetic anisotropy $K_{\mathrm{u}}$ induced by the FM/AFM exchange coupling. Our results will help promote the development of low-energy consumption magnetic device concepts for fast spin manipulation at room temperature.

\section{Results}

Description of ultrafast spin exchange-coupling torque. The observed ultrafast spin precession excitation is described by a modified Landau-Lifshitz-Gilbert (LLG) equation with an additional torque term:

$$
\frac{\partial \mathbf{M}}{\partial t}=-\gamma\left(\mathbf{M} \times \mathbf{H}_{\mathrm{eff}}\right)+\alpha \mathbf{M} \times \frac{\partial \mathbf{M}}{\partial t}+\tau_{\mathrm{ex}}(t),
$$

where $\gamma$ is gyromagnetic ratio, $\mathbf{M}$ is the magnetization, $\mathbf{H}_{\mathrm{eff}}$ is the effective magnetic field, $\alpha$ is the Gilbert damping constant, $\tau_{\mathrm{ex}}(t)=-\gamma\left(\mathbf{M} \times \Delta \mathbf{H}_{\mathrm{ex}}(t)\right)$ denotes the instant spin exchangecoupling torque, and $\Delta \mathbf{H}_{\mathrm{ex}}(t)$ is the change of FM-AFM exchange

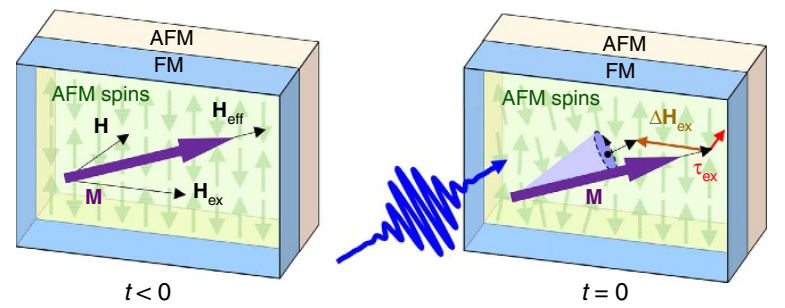

Figure 1 | Illustration of photo-excited spin exchange-coupling torque. At $t<0$, the magnetization $\mathbf{M}$ (purple arrow) in the Fe layer aligns along the effective field direction $\mathbf{H}_{\text {eff }}$ (black arrow). $\mathbf{H}$ denotes the external magnetic field, and $\mathbf{H}_{\mathrm{ex}}$ is the field established by FM-AFM exchange coupling. At $t=0$, the $400-\mathrm{nm}$ pump pulse (blue arrow) generates photo-excited carriers in the $\mathrm{CoO}$ layer, which leads to the reorientation of AFM spins (green arrows). This modifies the exchange coupling, $\Delta \mathbf{H}_{\mathrm{ex}}$ (brown arrow), causing a change of the effective field direction. Then the exchange-coupling torque $\tau_{\mathrm{ex}}$ (red arrow) forms, which triggers the precession of $\mathbf{M}$ field $\mathbf{H}_{\mathrm{ex}}$ by modulation of uniaxial exchange anisotropy $K_{\mathrm{u}}$, as discussed further below. The geometry of instant spin exchange-coupling torque $\tau_{\mathrm{ex}}(t)$ is illustrated in Fig. 1 .

Structure and static magnetic properties. The $\mathrm{Fe} / \mathrm{CoO}$ and $\mathrm{Fe}$ thin films are deposited on $\mathrm{MgO}(001)$ substrates by molecular beam epitaxy (MBE) at room temperature (see Methods) ${ }^{7}$. The thickness of $\mathrm{CoO}$ and Fe layers are $3 \mathrm{~nm}$ and $4 \mathrm{~nm}$, respectively. All of the samples are covered with a $3-\mathrm{nm}$-thick $\mathrm{MgO}$ protection layer, and the epitaxial relation is $\mathrm{CoO}[110] / / \mathrm{Fe}[100]$. Reflection high-energy electron diffraction patterns reveal the high-quality, epitaxial growth of the $\mathrm{CoO}$ and $\mathrm{Fe}$ films (see Supplementary Fig. 1). Longitudinal MOKE measurements are carried out with external magnetic field applied along the Fe [100] and [010] directions (Fig. 2a, right). The easy-axis hysteresis loops show perfect squareness, indicating the single domain of the Fe film (see Supplementary Fig. 2 and Supplementary Note 1).

Observation of ultrafast spin exchange-coupling torque. Coherent spin precessions in the Fe layers are investigated by pump-probe TRMOKE (see Methods) in a canted magnetization configuration where the magnetic field $(\mathbf{H})$ is applied along $\mathrm{Fe}$ [110] direction, as depicted in Fig. 2a. In equilibrium, the magnetization is along an effective field $\mathbf{H}_{\text {eff, }}$, which is the sum of $\mathbf{H}$, the demagnetizing field $\mathbf{H}_{\mathrm{d}}$ and the anisotropy field $\mathbf{H}_{\mathrm{a}}$. The incident pump pulses induce a transient field $\mathbf{H}_{\mathrm{tr}}$, and the magnetization $\mathbf{M}$ starts to precess around $\mathbf{H}_{\mathrm{tr}}$. When $\mathbf{H}_{\mathrm{tr}}$ has vanished, the vector $\mathbf{M}$ is away from its original equilibrium orientation along $\mathbf{H}_{\text {eff. }}$ Therefore, it starts to precess around $\mathbf{H}_{\text {eff }}$ as depicted in Fig. 2a. In general, the amplitude will show a resonance-type dependence on an external field, and the position and frequency of the resonance are determined by the external field and the magnetic parameters such as the values of the various anisotropy constants. Two strategies of pump are employed in the measurements to investigate the optical excitation mechanisms: First, the more intense pump pulses $(\lambda=800$ $\mathrm{nm}, 3.1 \mathrm{~mJ} \mathrm{~cm}^{-2}$ ) are used to modulate the FM order of Fe layer as shown in Fig. 2b (left) since the $\mathrm{CoO}$ layer is almost transparent to $800-\mathrm{nm}$ light ${ }^{11}$; Second, the weaker pump pulses $\left(\lambda=400 \mathrm{~nm}, 0.16 \mathrm{~mJ} \mathrm{~cm}^{-2}\right)$ are utilized to mainly affect the AFM order of $\mathrm{CoO}$ as depicted in Fig. 2b (right). All TRMOKE measurements are performed after field cooling the sample.

The TRMOKE result from $\mathrm{Fe} / \mathrm{MgO}$ heterostructure is displayed in Fig. 2c (black squares) with pump-pulse fluence $3.1 \mathrm{~mJ} \mathrm{~cm}{ }^{-2}$ and magnetic field $H=2 \mathrm{kOe}$ at room temperature. The sudden rise and decay of Kerr signal indicates the demagnetization process. Meanwhile, the magnetization starts to precess around the equilibrium direction in a damped circling way described by LLG equation ${ }^{12,13}$. The measured Kerr signal can be well-fitted by the following equation

$$
\theta_{k}=a \times \exp \left(-t / t_{0}\right)+A \times \exp (-t / \tau) \sin (2 \pi f t+\varphi),
$$

where parameters $A, \tau, f$ and $\varphi$ are the amplitude, magnetic relaxation time, frequency and initial phase of the magnetization precession mainly along the polar direction, respectively. Here, $a$ and $t_{0}$ are related to the background signal owing to the slow recovery of magnetization after fast demagnetization by the pump pulses, which happens mainly along the longitudinal direction.

Figure 2c (red circles) shows TRMOKE result from $\mathrm{Fe} / \mathrm{CoO}(3 \mathrm{~nm}) / \mathrm{MgO}$ structure with the same excitation condition as the measurement on $\mathrm{Fe} / \mathrm{MgO}$ (black squares). We note that the background amplitude $a$ remains unchanged, while the amplitude of coherent spin precession $A$ is enhanced. This shows that the AFM CoO layer improves the efficiency of optically excited coherent spin precession. The key finding here is that pronounced 
a

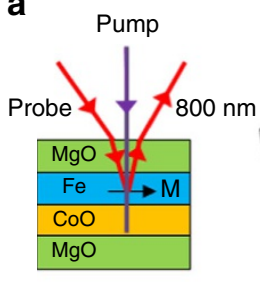

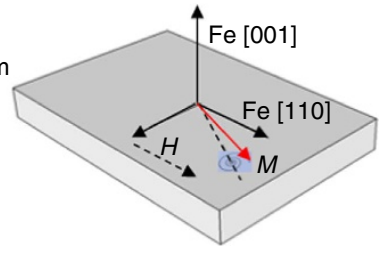

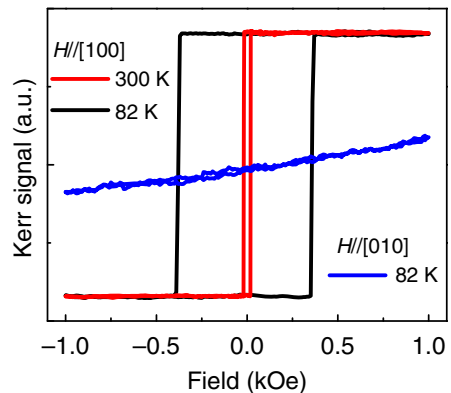

b

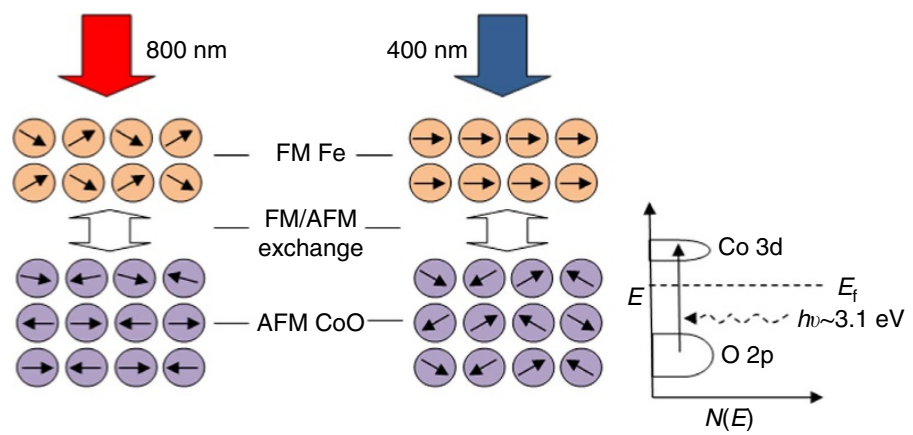

C

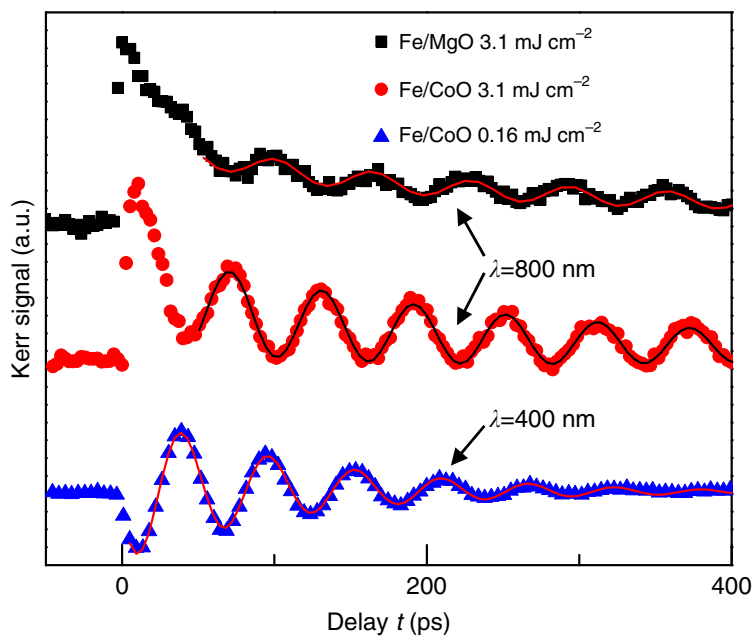

Figure 2 | Experimental design and observation of ultrafast exchange-coupling torque. Schematic of TRMOKE measurement geometry, depiction of magnetization precession and longitudinal hysteresis loops (a). Two pump strategies to optically excite the spin precession (b), where the black arrows represent the magnetic moments. Optical charge-transfer transition in $\mathrm{CoO}$ is depicted. TRMOKE results from Fe/MgO (black squares) and Fe/CoO (red circles) with pump-pulse intensity $3.1 \mathrm{~mJ} \mathrm{~cm}^{-2}$, and $\mathrm{Fe} / \mathrm{CoO}$ (blue triangles) with pump intensity $0.16 \mathrm{~mJ} \mathrm{~cm}^{-2}$ (c). The solid lines represent fits of equation (2).

spin precession is still observed with much lower pump-pulse energy $0.16 \mathrm{~mJ} \mathrm{~cm}^{-2}$ at $400-\mathrm{nm}$ wavelength, as shown in Fig. $2 \mathrm{c}$ (blue triangles). Moreover, the TRMOKE data reveal the absence of obvious demagnetization and slow recovery of $M$. The instant pronounced spin precession points towards an ultrafast nonthermal excitation process in the AFM CoO layer as depicted in Fig. 2b (right) and discussed further below. Furthermore, the magnetic relaxation time $\tau$ decreases from $330 \mathrm{ps}$ (red circles) to $100 \mathrm{ps}$ (blue triangles) in $\mathrm{Fe} / \mathrm{CoO}$ with $400-\mathrm{nm}$ pump pulses, which is desirable for fast switching. To determine the origin of the optical excitation mechanism, temperature and fielddependent TRMOKE measurements are carried out (Fig. 3).

Temperature- and field-dependent studies. Figure $3 \mathrm{a}$ presents TRMOKE results $(\lambda=400 \mathrm{~nm}, H=2 \mathrm{kOe})$ with pump-pulse intensity $0.16 \mathrm{~mJ} \mathrm{~cm}^{-2}$ at different temperatures $T$, where the absence of demagnetization peak is observed. Figure $3 \mathrm{~b}$ displays the precession frequency $f$ (red circles) and amplitude $A$ (black squares) as a function of $H$ at room temperature. The frequency $f$ is well-fitted with LLG equation (red curve) to derive the magnetic anisotropies (see Supplementary Note 2 and Supplementary Table 1$)^{8}$. The simulation of $A$ versus $H$ (black curve), where $A$ is assumed to be proportional to the equilibrium direction change by modulation of $K_{\mathrm{u}}\left(\Delta K_{\mathrm{u}}=165 \mathrm{Oe} \times M_{\mathrm{S}}\right)($ refs 14-16) (see Supplementary Note 3 ), agrees quite well with the experimental data. $M_{\mathrm{S}}$ is the saturation magnetization. Furthermore, $K_{\mathrm{u}}$ increases significantly with decreasing $T$, while $M_{\mathrm{S}}$ remains unchanged (see Supplementary Figure 3 and Supplementary Note 1), as shown by the blue triangles in Fig. 3c. The derived $K_{\mathrm{u}} / M_{\mathrm{S}}$ behaves similarly like $f$ (red circles and black squares) as a function of $T$. The FM-AFM exchange coupling establishes an extra preference of magnetization alignment in $\mathrm{Fe}$, where the FM spins favour perpendicular alignment with the 
a

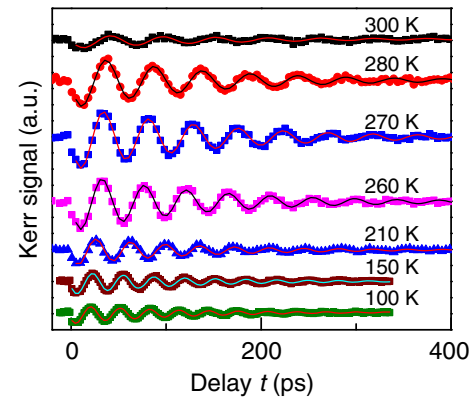

C

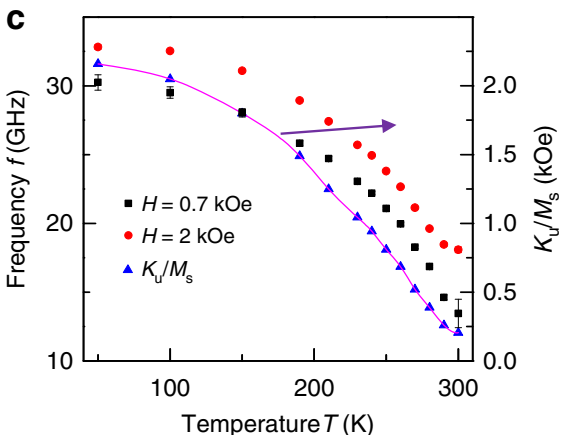

b
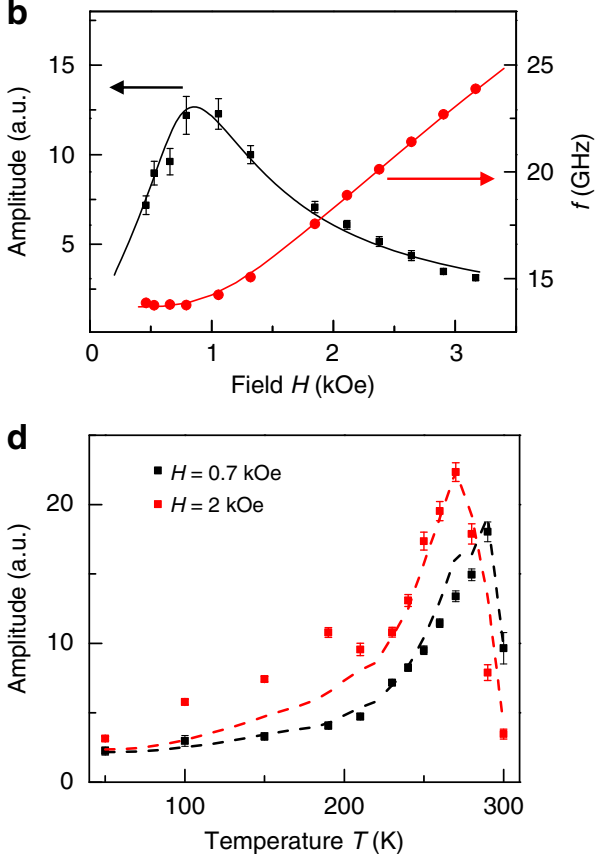

Figure 3 | Temperature and field-dependent TRMOKE studies. TRMOKE results from $\mathrm{Fe} / \mathrm{CoO}$ at different temperatures $T$ (a), where the solid lines are fits of equation (2). Spin precession amplitude $A$ (black squares) and frequency $f$ (red circles) as a function of $H$ (b). The solid lines are simulations. The arrows indicate the proper $\mathrm{y}$-axis for the different plots $(\mathbf{b}, \mathbf{c})$. Temperature dependence of $f$ for two different fields $(\mathbf{c})$. The derived $K_{\mathrm{u}} / M_{\mathrm{S}}$ is also plotted (blue triangles) and linked with spline cubic analysis fitting. Temperature dependence of $A$ for two different fields (d), where solid lines represent simulated results. Error bar of $A$ is estimated by the deviation of fitting with equation (2).
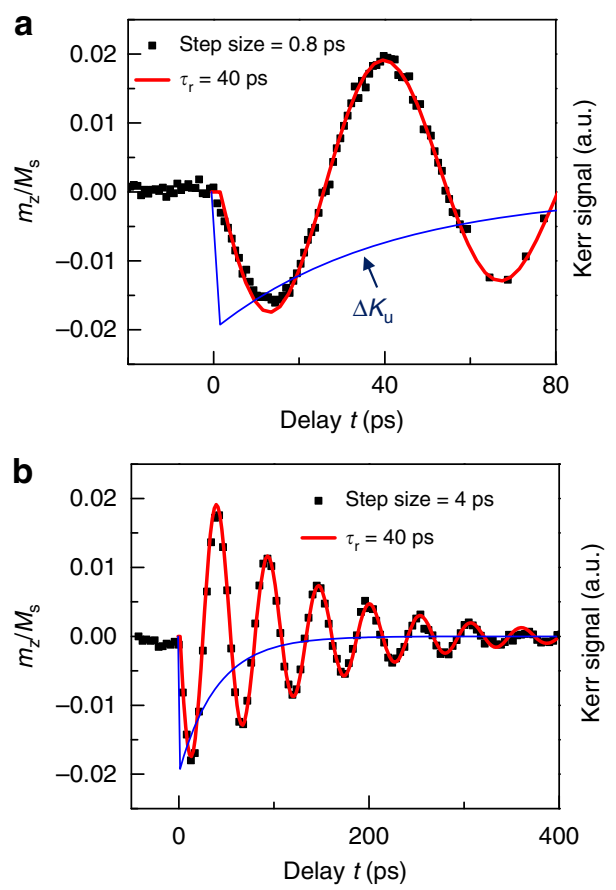

Figure 4 | Simulation of real-time magnetization precession. Measured Kerr signal change (black squares), simulated polar magnetization component (red curve) and assumed modulation of $K_{\mathrm{u}}$ (blue curves) as a function of time delay $t$ on short (a) and long (b) time scales.

frozen AFM spins due to the spin flop coupling between them. As the number of frozen AFM spins in CoO grows with lower $T$, the FM/AFM exchange coupling builds up, which leads to an increase of $K_{\mathrm{u}}$ and hence $f$ rises with lower $T$ as shown in Fig. 3c. Figure 3d displays the precession amplitude $A$ as a function of $T$ for different $H$. The amplitude $A$ peaks around $270 \mathrm{~K}(H=2 \mathrm{kOe})$ and $290 \mathrm{~K}(H=0.7 \mathrm{kOe})$, when $H$ is approximately equal to the anisotropy field $H_{\mathrm{a}}$. At higher temperatures, $A$ drops sharply similar to $K_{\mathrm{u}} / M_{\mathrm{S}}$, because the AFM order in $\mathrm{CoO}$ is greatly diminished above $270 \mathrm{~K}$, since the temperature is close to its Neel temperature $(\sim 290 \mathrm{~K})$ and the FM/AFM exchange coupling becomes weaker. Therefore the uniaxial anisotropy $K_{u}$ is small compared with $H$ when approaching room temperature. This behaviour is similar to the peak shown in Fig. $3 \mathrm{~b}$. As $T$ decreases, $K_{\mathrm{u}}$ enhances $H_{\mathrm{a}}$, which requires a stronger $H$ to compete with $H_{\mathrm{a}}$, hence the generated transient torque and amplitude $A$ decrease. As a result, the peak in $A$ shifts to lower $T$ and becomes broader at higher fields, which agrees well with the simulation based on the modulation of $K_{\mathrm{u}}$ (dashed curves). Therefore, the origin of this efficient excitation mechanism with $400-n m$ pump pulses has to be the modulation of magnetic anisotropy constant $K_{\mathrm{u}}$.

\section{Discussion}

The modulation of $K_{\mathrm{u}}$ also sheds light on the difference between the two optical excitation strategies, $800-\mathrm{nm}$ versus $400-\mathrm{nm}$ wavelength pump pulses. On one hand, the CoO layer is almost transparent to light with $\lambda=800 \mathrm{~nm}$, as its bandgap is $\sim 2.5 \mathrm{eV}$ (ref. 11). Therefore, the $800-\mathrm{nm}$ pump pulses mainly excite hot electrons in the FM Fe layer as depicted in Fig. 2b (left) which also modulate the FM-AFM exchange coupling and hence $K_{\mathrm{u}}$. This leads to improvement of spin precession excitation efficiency as compared with $\mathrm{Fe} / \mathrm{MgO}$ thin film (Fig. 2c). Ju. et al. ${ }^{17}$ reported that in the exchange-coupled $\mathrm{NiFe} / \mathrm{NiO}$ bilayer the photo-excited hot electrons in the FM layer partially diffuse to the interface and slightly modulate the interface AFM order. The authors called this process an 'ultrafast unpinning of the exchange bias' ${ }^{17}$. The authors also note that the bulk magnetic structure of $\mathrm{NiO}$ is frozen ${ }^{17}$. This is markedly different from the phenomenon observed here, which we assume here to modulate the AFM order in the $\mathrm{CoO}$ layer. 
Photon-induced interband transitions occur in bulk $\mathrm{CoO}$ at $\lambda=455 \mathrm{~nm}(2.73 \mathrm{eV})$ (see Supplementary Note 4). Hence the near-gap, 400-nm pump photons excite charge-transfer transitions from $\mathrm{O} 2 \mathrm{p}$-band to Co $3 \mathrm{~d}$-band that is partially occupied with minority spins, as depicted in Fig. 2b (ref. 18). This promotes the nearest-neighbour FM exchange interaction ${ }^{19}$, which modulates the AFM order in the $\mathrm{CoO}$ layer. Similarly, Duong et al. ${ }^{20}$ reported ultrafast manipulation of AFM order in $\mathrm{NiO}$. Photoexcitation of $\mathrm{NiO}$ leads to ultrafast reorientation of $\mathrm{Ni}^{2+}$ spins due to change of the magnetic anisotropy ${ }^{20}$. The variance of AFM order in $\mathrm{CoO}$ layer notably modulates the FM/ AFM exchange coupling and $K_{\mathrm{u}}$, since the coupling between Fe and $\mathrm{CoO}$ is a long-range interaction ${ }^{21}$. This leads to a change of the effective field direction, as depicted in Fig. 1, causing a transient torque on the ferromagnetic magnetization and thus leads to significant enhancement of the excited FM spin precession. We call this process an 'ultrafast spin exchangecoupling torque', which is markedly different from the ultrafast unpinning process due to hot electrons in the FM layer. For the ultrafast spin exchange-coupling torque, the carrier excitation is instant on photoexcitation, thus the AFM modulation is fast. In addition, the exchange interaction between Fe FM spins and $\mathrm{CoO}$ AFM spins are very strong and the modulation of this exchange interaction is fast. Among the magnetic interactions, the spin exchange-coupling interaction has the largest energy and thus the shortest time scale. This process is also repeatable many times without any thermally induced degradation. Larger magnetization precession may be obtained with shorter wavelengths, thereby enhancing (reducing) the absorption in the $\mathrm{CoO}(\mathrm{Fe})$ layer, which will also lower the thermal load (larger heat capacity of $\mathrm{CoO}$ ) allowing the application of higher pump-pulse power.

To investigate the duration of this fast FM-AFM exchange torque, simulations of real-time magnetization precession are carried out from LLG equation. The observed pronounced coherent spin precession starts right at $t=0$, indicating a sudden change of equilibrium direction caused by $\Delta K_{\mathrm{u}}$. We assume that the reduction of $K_{\mathrm{u}}\left(165 \mathrm{Oe} \times M_{\mathrm{S}}\right)$ happens instantly at $t=0$, followed by an exponential recovery process with time constant $\tau_{\mathrm{r}}$, as shown by the blue curve in Fig. 4 . With $\tau_{\mathrm{r}}=40 \mathrm{ps}$, the simulated time evolution of magnetization (red curve) agrees quite well with the probed Kerr signal (black dots), since the TRMOKE signal is proportional to the polar component of magnetization precession. Shorter or longer $\tau_{\mathrm{r}}$ leads to mismatch in the oscillation phase (See Supplementary Fig. 4). The fast recovery from photo-excited transitions might be due to the strong carrier-phonon interaction, which results in non-radiative and phonon-assisted carrier recombination ${ }^{22}$. Moreover, the recovery time of the instant photo-induced exchange-coupling torque is much faster than the cooling time from demagnetization, which can promote novel device concepts for fast spin manipulation.

In summary, the efficiency of spin precession excitation by laser pulses is significantly improved by inserting an AFM CoO layer in $\mathrm{Fe} / \mathrm{MgO}$ heterostructure, which establishes the uniaxial magnetic anisotropy $K_{\mathrm{u}}$ along Fe [100] direction. The modulation of $K_{\mathrm{u}}$ by laser pump pulses generates a fast exchange-coupling torque to the FM Fe magnetization. The transient torque is enhanced at temperatures where $K_{\mathrm{u}}$ varies significantly and with external magnetic fields comparable to the magnetic anisotropy fields. The excitation is much more efficient with $400-\mathrm{nm}$ pump pulses via photo-excited charge-transfer processes in AFM CoO layer than modulating the FM order of $\mathrm{Fe}$ via generation of hot electrons with 800-nm-wavelength pulses. The recovery time of the exchange-coupling torque is $\sim 40 \mathrm{ps}$, much faster than the cooling time from demagnetization. Our results will help promote the development of low-energy consumption magnetic device concepts for fast spin manipulation at room temperature.

\section{Methods}

Sample fabrication. The $\mathrm{Fe} / \mathrm{CoO}$ thin films are grown on the $\mathrm{MgO}(001)$ substrate in an ultrahigh vacuum chamber with $\mathrm{MBE}$. The $\mathrm{MgO}(001)$ substrate with a miscut angle $<0.5^{\circ}$ is prepared by annealing at $600^{\circ} \mathrm{C}$ for $30 \mathrm{~min}$. The AFM CoO thin films are grown by a reactive deposition of Co under an oxygen pressure of $2 \times 10^{-6}$ torr. A 4 -nm-thick Fe film is then grown epitaxially on top of the $\mathrm{CoO}$ film at room temperature. All samples are covered with a 3-nm-thick $\mathrm{MgO}$ protection layer. As a control group, $\mathrm{Fe}$ thin films are grown directly on the $\mathrm{MgO}$ substrate with MBE at room temperature.

MOKE measurements. In the longitudinal MOKE studies, we measured the FM magnetization of Fe layer by irradiating the sample with p-polarized light and detecting the s-component of the reflected light with a photodiode. The external magnetic field is applied in-plane along the $\mathrm{Fe}$ [100] or [010] directions from $80 \mathrm{~K}$ to above room temperature.

Time-resolved MOKE measurements. We performed TRMOKE measurements in a pump-probe set-up, where the intensity ratio of the pump to probe pulses is set to be about 6:1. The probe $(\lambda=800 \mathrm{~nm})$ utilizes the MOKE technique with crossed polarizers and incident angle $\sim 40^{\circ}$ to investigate the transient magnetic state change along longitudinal and polar directions. The intense pump pulses $\left(3.1 \mathrm{~mJ} \mathrm{~cm}^{-2}\right)$ are generated by a Ti/sapphire amplifier laser system delivering 150 -fs pulses at $800-\mathrm{nm}$ wavelength with a repetition rate of $1 \mathrm{kHz}$. The $400-\mathrm{nm}$ pump pulses $\left(0.16 \mathrm{~mJ} \mathrm{~cm}^{-2}\right)$ are generated by frequency-doubling the $800-\mathrm{nm}$ pulses (200 fs) from a $250-\mathrm{kHz}$ Ti/sapphire laser system in a beta barium borate (BBO) crystal.

Data analysis and simulations. The TRMOKE raw data is fitted using the built-in non-linear-fit function of Origin 8.5 software with expression programmed as equation (2). The uncertainties of $A$ and $f$ are provided by the software from least squares fitting. Least square methods are also programmed with Matlab 2014 and used to fit the change of $f$ and $A$ as functions of $H$ with Supplementary Equations 1 and 3 (see Supplementary Note 3), where the uncertainties of anisotropy fields are estimated through error propagation method. To simulate the real-time magnetization precession in Fig. 4, LLG equation is written numerically in Supplementary Equations 4 and 5 , with very small time interval $\Delta t=0.2 \mathrm{ps}$ (see Supplementary Note 5). The evolution of $y$ and $z$ magnetization components with time is derived through iteration, which is programmed with Matlab 2014.

\section{References}

1. Stamm, C. et al. Femtosecond modification of electron localization and transfer of angular momentum in nickel. Nat. Mater. 6, 740-743 (2007).

2. Koopmans, B. et al. Explaining the paradoxical diversity of ultrafast laser-induced demagnetization. Nat. Mater. 9, 259-265 (2010).

3. Rudolf, D. et al. Ultrafast magnetization enhancement in metallic multilayers driven by superdiffusive spin current. Nat. Commun. 3, 1037 (2012).

4. Choi, G.-M., Min, B.-C., Lee, K.-J. \& Cahill, D. G. Spin current generated by thermally driven ultrafast demagnetization. Nat. Commun. 5, 4334 (2014).

5. Nemec, P. et al. Experimental observation of the optical spin transfer torque. Nat. Phys. 8, 411-415 (2012)

6. Tesarova, N. et al. Experimental observation of the optical spin-orbit torque. Nat. Photon. 7, 492-498 (2013).

7. Cao, W. N. et al. Temperature-dependent magnetic anisotropies in epitaxial $\mathrm{Fe} / \mathrm{CoO} / \mathrm{MgO}(001)$ system studied by the planar Hall effect. Appl. Phys. Lett. 98, 262506 (2011)

8. Fan, Y. et al. Photoinduced spin angular momentum transfer into an antiferromagnetic insulator. Phys.Rev. B 89, 094428 (2014).

9. Kimel, A. V., Kirilyuk, A., Usachev, P. A., Pisarev, R. V. \& Balbashov, A. M. Ultrafast non-thermal control of magnetization by instantaneous photomagnetic pulses. Nature 435, 655-657 (2005)

10. Kimel, A. V., Kirilyuk, A., Tsvetkov, A., Pisarev, R. V. \& Rasing, T. Laser-induced ultrafast spin reorientation in the antiferromagnet $\mathrm{TmFeO} 3$. Nature 429, 850-853 (2004).

11. Kant, C. et al. Optical spectroscopy in CoO: Phononic, electric, and magnetic excitation spectrum within the charge-transfer gap. Phys. Rev. B 78, 245103 (2008).

12. Landau, L. \& Lifshitz, E. On the theory of the dispersion of magnetic permeability in ferromagnetic bodies. Phys. Z. Sowjetunion 8, 153 (1935).

13. Gilbert, T. L. A phenomenological theory of damping in ferromagnetic materials. IEEE Trans. Magn. 40, 3443-3449 (2004).

14. Liu, Y. et al. Optically induced magnetization dynamics and variation of damping parameter in epitaxial Co2MnSi Heusler alloy films. Phys. Rev. B 81, $094402(2010)$. 
15. Hansteen, F., Kimel, A., Kirilyuk, A. \& Rasing, T. Femtosecond photomagnetic switching of spins in ferrimagnetic garnet films. Phys. Rev. Lett. 95, 047402 (2005).

16. Kirilyuk, A., Kimel, A. V. \& Rasing, T. Ultrafast optical manipulation of magnetic order. Rev. Modern Phys. 82, 2731-2784 (2010).

17. Ju, G., Nurmikko, A. V., Farrow, R. F. C., Marks, R. F. \& Carey, M. J. Ultrafast time resolved photoinduced magnetization rotation in a ferromagnetic/ antiferromagnetic exchange coupled system. Phys. Rev. Lett. 82, 3705-3708 (1999).

18. Jiang, C.-M. et al. Characterization of photo-induced charge transfer and hot carrier relaxation pathways in spinel cobalt oxide (Co3O4). J. Phys. Chem. C 118, 22774-22784 (2014).

19. Deng, H.-X., Li, J., Li, S.-S., Xia, J.-B. \& Walsh, A. Origin of antiferromagnetism in CoO: A density functional theory study. Appl. Phys. Lett. 96, 162508 (2010).

20. Duong, N. P., Satoh, T. \& Fiebig, M. Ultrafast manipulation of antiferromagnetism of NiO. Phys. Rev. Lett. 93, 117402 (2004).

21. Valev, V., Gruyters, M., Kirilyuk, A. \& Rasing, T. Direct observation of exchange bias related uncompensated spins at the $\mathrm{CoO} / \mathrm{Cu}$ interface. Phys. Rev. Lett. 96, 067206 (2006).

22. Volkov, V. V., Wang, Z. L. \& Zou, B. S. Carrier recombination in clusters of NiO. Chem. Phys. Lett. 337, 117-124 (2001).

\section{Acknowledgements}

The TRMOKE experiments, data analysis, simulations and discussions performed at the College of William and Mary were sponsored by the DOE through Grant No. DEFG02-04ER46127. The work at the Department of Physics, Fudan University, was supported by the National Key Basic Research Program (No. 2015CB921401, No. 2011CB921801) and the National Science Foundation (No. 11434003, No. 11474066) of China. The work at the Department of Optical Science and Engineering, Fudan
University, was supported by the NSFC with Grants No. 61222407 and No. 51371052 and the National Key Basic Research Program (No. 2015CB921403).

\section{Author contributions}

X.M., Y.Z.W., H.B.Z. and G.L. conceived the experiments. Q.L., J.Z. and Y.Y. prepared the samples and carried out characterizations using MOKE, RHEED and SQUID measurements. X.M. and F.F. performed the TRMOKE experiments. X.M. performed the data analysis and simulations. X.M., Y.Z.W., H.B.Z. and G.L. wrote the manuscript.

\section{Additional information}

Supplementary Information accompanies this paper at http://www.nature.com/ naturecommunications

Competing financial interests: The authors declare no competing financial interests.

Reprints and permission information is available online at http://npg.nature.com/ reprintsandpermissions/

How to cite this article: Ma, X. et al. Ultrafast spin exchange-coupling torque via photoexcited charge-transfer processes. Nat. Commun. 6:8800 doi: 10.1038/ncomms 9800 (2015).

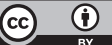

This work is licensed under a Creative Commons Attribution 4.0 International License. The images or other third party material in this article are included in the article's Creative Commons license, unless indicated otherwise in the credit line; if the material is not included under the Creative Commons license, users will need to obtain permission from the license holder to reproduce the material. To view a copy of this license, visit http://creativecommons.org/licenses/by/4.0/ 